\title{
Understanding the variability of magnetic storms caused by ICMEs
}

\author{
Remi Benacquista $^{1}$, Sandrine Rochel ${ }^{1}$, and Guy Rolland ${ }^{2}$ \\ ${ }^{1}$ Departement environnement spatial, ONERA, Toulouse, France \\ ${ }^{2}$ CNES, The French Space Agency, Toulouse, France \\ Correspondence to: Remi Benacquista (remi.benacquista@onera.fr) \\ Received: 6 June 2016 - Revised: 3 January 2017 - Accepted: 11 January 2017 - Published: 30 January 2017
}

\begin{abstract}
In this paper, we study the dynamics of magnetic storms due to interplanetary coronal mass ejections (ICMEs). We used multi-epoch superposed epoch analyses (SEAs) with a choice of epoch times based on the structure of the events. By sorting the events with respect to simple largescale features (presence of a shock, magnetic structure, polarity of magnetic clouds), this method provides an original insight into understanding the variability of magnetic storm dynamics. Our results show the necessity of seeing ICMEs and their preceding sheaths as a whole since each substructure impacts the other and has an effect on its geoeffectiveness. It is shown that the presence of a shock drives the geoeffectiveness of the sheaths, while both the shock and the magnetic structure impact the geoeffectiveness of the ICMEs. In addition, we showed that the ambient solar wind characteristics are not the same for ejecta and magnetic clouds (MCs). The ambient solar wind upstream magnetic clouds are quieter than upstream ejecta and particularly slower. We also focused on the polarity of magnetic clouds since it drives not only their geoeffectiveness but also their temporal dynamics. South-north magnetic clouds (SN-MCs) and north-south magnetic clouds (NS-MCs) show no difference in geoeffectiveness for our sample of events. Lastly, since it is wellknown that sequences of events can possibly induce strong magnetic storms, such sequences have been studied using superposed epoch analysis (SEA) for the first time. We found that these sequences of ICMEs are very usual and concern about $40 \%$ of the ICMEs. Furthermore, they cause much more intense magnetic storms than isolated events do.
\end{abstract}

Keywords. Magnetospheric physics (solar windmagnetosphere interactions)

\section{Introduction}

ICMEs (interplanetary coronal mass ejections) are the interplanetary manifestation of CMEs occurring at the sun's surface. In the interplanetary medium, most ICMEs propagate faster than the ambient solar wind. This results in a disturbed region upstream form each ICME caused by an accumulation of plasma and magnetic field as an ICME propagates and expands in the interplanetary medium. If the speed of the ICME is high enough, a shock is formed at the forward limit of the disturbed region, which is then called a "sheath".

ICMEs can be divided into two subsets according to their magnetic structure (Burlaga, 2001): magnetic clouds and complex ejecta. On the one hand, magnetic clouds (MCs) are basically ICMEs with a magnetic flux rope structure (Burlaga et al., 1981). This results in smooth variations in the magnetic field inside the $\mathrm{MC}$, which can thus be expressed by a polarity indicating the orientation of the $z$ component of the magnetic field. On the other hand, ICMEs can be complex ejecta, which do not show such a structure. For the last decades, much effort has been made to understand ICMEs, and more particularly, the MC subset. Cane and Richardson (2003) show that the number of ICMEs depend directly on the solar cycle, being higher near solar maximum. Conversely, the MC rate is higher near the solar minimum (Richardson and Cane, 2004). More precisely, Mulligan et al. (1998) and Li and Luhmann (2004) show that the distribution of the polarity of the MCs also depends on the Hale 22-year cycle. The structures of ICMEs have also been extensively studied (Tsurutani et al., 2004; Zurbuchen and Richardson, 2006; Kilpua et al., 2011, 2013) and many studies looked for correlations between several solar wind (SW) parameters in ICMEs (e.g., Gonzalez et al., 1998; Owens and Cargill, 2002; Echer et al., 2005; Gopalswamy, 2008). 
Geomagnetic indices are commonly used as monitors of the Earth's magnetic field disturbances caused, among others, by ICMEs. There have been many attempts to link the geomagnetic indices and the characteristics of the ICMEs, such as the speed, the apparent width, the source position, and the magnetic field (e.g., Dumbović et al., 2015). It is well established that both ICMEs (or MCs) and the sheaths preceding them can deeply disturb the magnetosphere, causing magnetic storms (Tsurutani et al., 1988; Crooker, 2000; Huttunen et al., 2002; Wu and Lepping, 2002; Huttunen and Koskinen, 2004; Huttunen et al., 2005; Pulkkinen et al., 2007; Richardson and Cane, 2012; Hietala et al., 2014; Yermolaev et al., 2015). Huttunen and Koskinen (2004) show that sheaths have a higher impact on auroral regions (measured by the index AE), while ICMEs disturb the ring current more strongly (measured by Dst or SYM-H). Finally, since the polarity is critical for the observed southward magnetic field profile within an ICME, it is commonly considered as a key parameter for understanding the geoeffectiveness (ability to cause some geomagnetic disturbances) of magnetic clouds (Fenrich and Luhmann, 1998; Li and Luhmann, 2004; Huttunen et al., 2005; Kilpua et al., 2012).

During the last decade, many case studies have been made to show that some sequences of events could cause extreme magnetic storms (e.g., Wang et al., 2003; Skoug et al., 2004; Veselovsky et al., 2004; Liu et al., 2014). Other statistical studies confirm that these sequences of events are more likely to produce strong magnetic storms. Eselevich and Fainshtein (1993) considered 100 magnetic storms between 1973 and 1983. They showed that in the case of non-isolated events, the magnetic storm contains two peaks, the second one being almost systematically higher (in amplitude) than the first one. Echer and Gonzalez (2004) studied a large number of solar wind structures ( 574 shocks, 946 sector boundary crossings, $149 \mathrm{MCs})$. They defined the geoeffectiveness of the event relative to the minimum of Dst. They showed that MCs are the most geoeffective solar wind structures and that their geoeffectiveness is increased when the magnetic clouds follow other structures (e.g., shocks). Farrugia et al. (2006) studied the 16 events with the highest energy deposition (using the Akasofu parameter $\epsilon$, Akasofu (1981)). They underlined that six of them are related to ICMEs interacting with each other and thus that sequences of such events are a source of geoeffectiveness.

Among others statistical methods, superposed epoch analysis (hereafter SEA) are commonly used in the study of sun-Earth interactions (e.g., Lyatsky and Tan, 2003; Lavraud et al., 2006; Denton et al., 2006; Borovsky and Denton, 2010). This method allows the determination of the average variations in a parameter (SW parameter, geomagnetic index, flux in the radiation belts) around a reference instant called epoch time. Ilie et al. (2008) pointed out the relevance of the choice of the epoch time (see also Yermolaev et al. (2010) and references therein), which is set most of the time as the maximum of the magnetic storm, defined as the minimum of the Dst index (e.g., Miyoshi and Kataoka, 2005; Zhang et al., 2006; Turner et al., 2009; Guo et al., 2011). Yermolaev et al. (2007) and Yermolaev et al. (2010) separately considered the sheaths and the ICMEs and compared their efficiencies (which are the effectivities relative to the "inputs"). They concluded that the sheaths are the most geoefficient structures before the ejecta. They also showed that MCs are less geoefficient despite the fact that they still play a role in storm generation (Yermolaev et al., 2012). However, none of these studies employed the SEA method to distinguish MCs with respect to their polarity.

In this paper, we use superposed epoch analysis (SEA) to determine the average characteristics of ICMEs as well as their impact on the magnetosphere. While most of the studies based on the SEA method use an epoch time related to the reaction of the magnetosphere (minimum of Dst), we chose to consider epoch times related to the structure of the events (onset of the sheath, onset and end of the ICME) and to compute multi-epoch SEA (see also Kilpua et al. (2015), where a similar method was used to study how radiation belt electron fluxes respond to different solar wind driver structures, or Yermolaev et al. (2015)). Such a method allows us to observe both the intensity and the temporal profile of the magnetic storms during different parts of the event. More particularly, it is possible to study the respective contributions of shocks, compressed regions, and ICMEs while the magnetosphere is disturbed. Furthermore, by making subsets of events with respect to large-scale features (presences of a shock, structure of the ICME, sequences of events), it is possible to determine the respective contributions of such features.

Hereafter, for clarity, we call every disturbed region upstream of ICMEs a sheath (delimited by a shock or not). We also refer to the whole (shock)-sheath-ICME structure as an event. Finally, the two subsets of ICME are either magnetic clouds (MCs) or ejecta (rather than complex ejecta).

\section{Data and method}

\subsection{List of events}

Our list is based on the merger of two lists: an ICMEs list and a magnetic clouds list. The first one was provided by Mitsakou and Moussas (2014b) and corrected a few months later (Mitsakou and Moussas, 2014a). By studying the evolution of the magnetic field, the proton density, and the temperature, the authors identified 325 ICMEs in the 1 min OMNI database in the 1996-2008 time period. For each event, the onset and the end date of the ICME are provided as well as the onset time of the sheath associated with the ICMEs. This allows us to separately study the effects of both sheaths and ICMEs. The authors also indicate if the sheath is associated with a shock but do not specify if the ICME is a MC or not. The second list provides the onset and the end date of MCs using an automatic detection method (Lepping et al., 2005, 


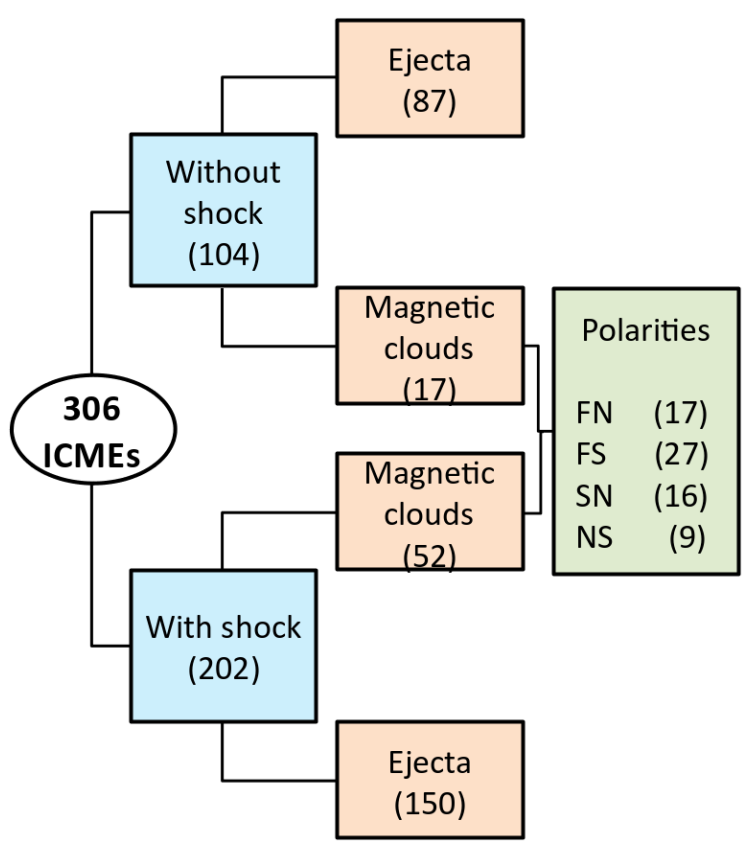

Figure 1. Organigram summarizing the distribution of ICMEs according to the presence of a shock upstream of the ICME (in blue), the magnetic structure of the event (in orange), and the polarity of magnetic clouds (in green). The numbers in parentheses indicate the number of events in each group. The different polarities are defined in Sect. 4.

2006). In this way, $101 \mathrm{MCs}$ are identified in the 1996-2008 period and the lists are in good agreement with the known proportion of magnetic clouds among ICMEs (one-third, see Gosling et al. (1990)). Then, for each ICME we determine if it is a magnetic cloud or not. To do so, the two lists were compared and an ICME is considered as a MC if there is a MC for which the dates correspond. Thus, we exclude the following from our study: ICMEs and MCs for which the association is unclear, magnetic clouds with no ICME associated, and ICMEs with two magnetic clouds. Figure 1 shows a chart summarizing our classification. Our list involves a total of 306 events. Two out of three ICMEs are preceded by a shock and $23 \%$ are magnetic clouds. Notice that the list involves only 17 magnetic clouds without shocks.

\subsection{Data set}

We used the OMNI database for both solar wind parameters and geomagnetic indices (King and Papitashvili, 2005). Here we study three solar wind parameters: the magnetic field, the dynamic pressure, and the speed. They are provided by $1 \mathrm{~min}$ OMNI database and averaged to a $5 \mathrm{~min}$ resolution. The geomagnetic indices are calculated using ground magnetometers, which are located at the feet of magnetic field lines crossing different regions of the magnetosphere. They are calculated by estimating the variations in the magnitude of the horizontal component of the local magnetic field, which are directly linked to the variations in the geomagnetic current intensity (Menvielle et al., 2011). We used two geomagnetic indices : AE and SYM-H. The AE index is calculated from magnetometers located in the auroral zone and provides information on the intensity of the electrojets, which are connected to the tail and the magnetopause currents (Ganushkina et al., 2015). The SYM-H index was used to estimate the ring current variations by using magnetometers close to the magnetic equator. It can be viewed as a high-resolution Dst (Wanliss and Showalter, 2006). It has been shown that other currents such as the magnetopause current affect SYM-H. The eastward magnetopause current induces positive magnetic disturbance when its intensity increases (then Dst or SYM-H increase). Conversely, the ring current is westward and thus tends to make SYM-H and Dst decrease. Using these two $5 \mathrm{~min}$ indices allows us to have a global view of the current system and to observe sharp changes due to discontinuities such as shocks. It should also be pointed out that Mitsakou and Moussas (2014b) also used the OMNI database (with the $1 \mathrm{~min}$ resolution) to determine their times of events; therefore, there is no possible delay between the times of the list and the variations of the parameters.

\subsection{Method}

A superposed epoch analysis (SEA) method was used. It determines the average behavior of a given parameter for a given list of events around a so-called epoch time. While the overall tendency is to set the reference date as the minimum of Dst (or SYM-H), we chose to study the ICME impact on the magnetosphere from the ICME main boundaries: the onset of the sheath, the onset of the ICME, and the end of the ICME (see Mitsakou and Moussas (2014b)). We therefore performed a multi-epoch SEA, where these three times are considered as epoch times (Yermolaev et al., 2010, 2015; Kilpua et al., 2015). To do so, the time spans between the epoch times are normalized and set equal to the average duration of each substructure. Thus, the durations of all sheaths (time between the first and second epoch time) are set equal to 0.38 days and the durations of all ICMEs (between the second and the third epoch time) are set equal to 1.02 days, where 0.38 and 1.02 days are the average durations of the sheaths and ICMEs. This method avoids confusing the effect of the different substructures and allows us to observe the dynamics of the magnetic storm during the event crossing.

\section{Solar wind and geomagnetic index characteristics for the entire list of events}

\subsection{Results}

Figure 2 shows multi-epoch SEA of three solar wind parameters (the dynamic pressure $P$, the magnetic field magnitude $B$, and the speed $V$ ) and two geomagnetic indices (SYM$\mathrm{H}$ and $\mathrm{AE}$ ). The three vertical lines indicate the three epoch 

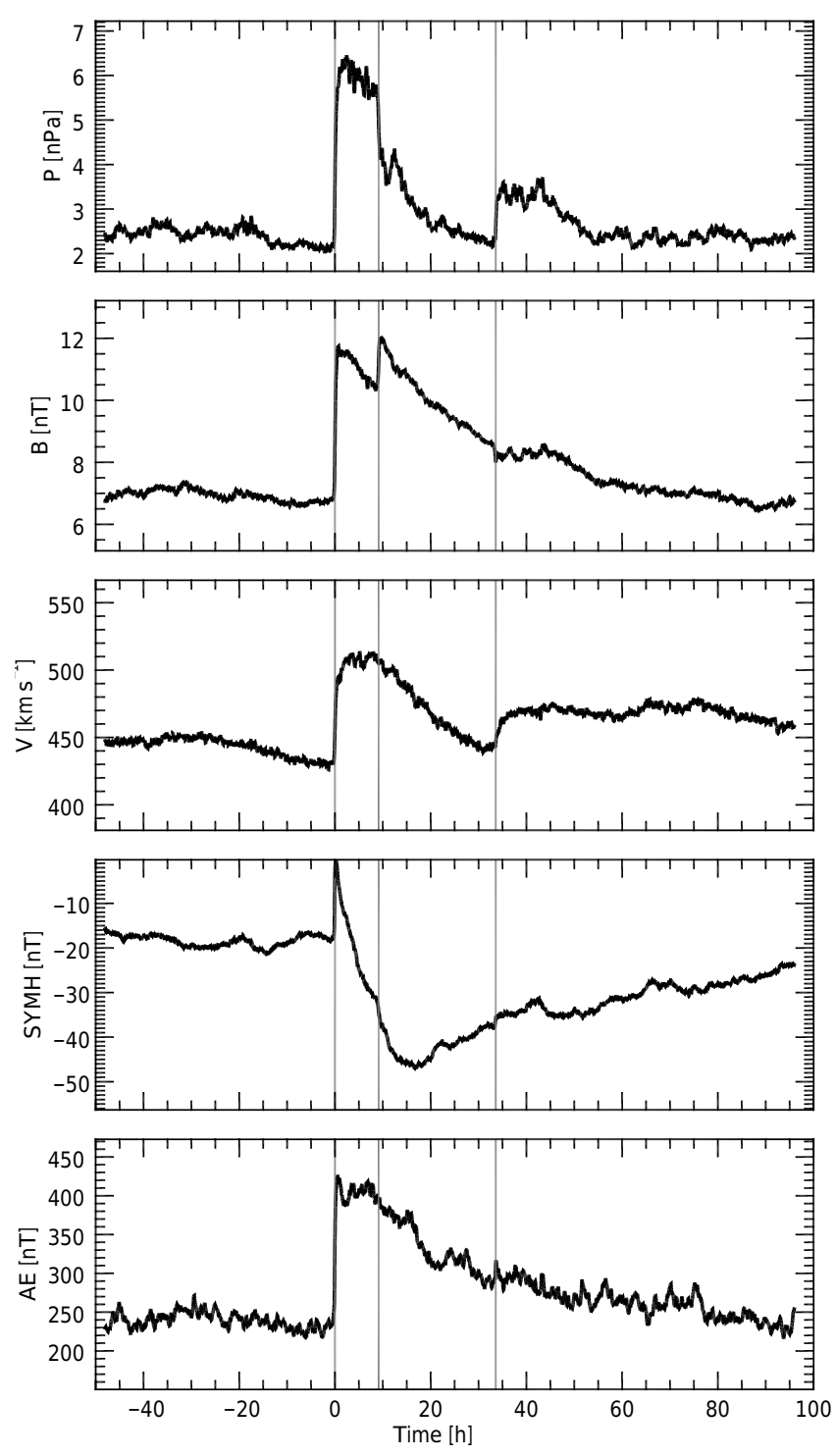

Figure 2. SEAs of three $\mathrm{SW}$ parameters $(B, P, V)$ and two geomagnetic indices (SYM-H and AE) for 306 ICMEs between 1996 and 2008. The three vertical lines are the epoch times indicating (respectively from left to right) the onset of the sheath, the onset of the ICME, and the end of the ICME.

times (onset of the sheath, onset of the ICME, end of the ICME). All parameters are displayed from 2 days before, to 4 days after the onset of the sheath.

The solar wind parameters displayed in Fig. 2 show characteristic signatures for each ICME structure. First, strong increases in all shown parameters indicate the arrival of the shock or the beginning of the sheath. Then both substructures (sheath and ICME) display some different characteristics. During the sheath, all parameters remain high. During the ICME, $P$ is low and $B$ and $V$ decrease progressively. The behavior of the solar wind parameters after the third epoch time should also be noticed. All three parameters dis- play values higher than before the first epoch time either for a few hours (or $P$ and $B$ ) or for a few days $(V)$. This will be discussed in Sect. 6.

The impact of such events on the magnetosphere is measured by the two geomagnetic indices in Fig. 2. At the first epoch time, the sharp increase in all solar wind parameters induces a strong reaction, with both of the indices increasing strongly. During the sheath, disturbances measured by AE remain high, while SYM-H decreases gradually. Then, from the onset of the ICME until its ends, the averaged AE seems to decrease progressively. In the meantime, SYM-H keeps decreasing and reaches its maximal amplitude during the first half of the ICME.

\subsection{Discussion}

While the strong increase in $\mathrm{AE}$ at the first epoch time indicates a quick increase in the intensity of the auroral electrojets, the meaning of the variations of SYM-H is less straightforward to interpret. It is well-known that the magnetopause current is related to the root square of the pressure (Burton et al., 1975). Thus, we believe that the sharp increase (decrease) in SYM-H at the first (second) epoch time, occurring at the same time as the similar increase (decrease) in pressure, is due also to an increase (decrease) in the magnetopause current and not only to a potential decrease (increase) in the ring current. Then, during the event crossing our results show that both the sheaths and the ICMEs disturb the current systems. In particular, it should be underlined that the current systems are still undergoing the disturbances caused by the sheath when the ICME impacts it. SEAs also indicate that $\mathrm{AE}$ is more disturbed by the sheath than by the ICME. This is consistent with previous study (e.g., Huttunen and Koskinen, 2004). However, one should keep in mind that because of the movement of the auroral oval, the AE stations may underestimate magnetic perturbations in the case of strong magnetic storms.

The maximal average amplitude observed on the geomagnetic indices are quite small $\left(\mathrm{SYM}-\mathrm{H}_{\min }=-47 \mathrm{nT}\right)$, which seems surprisingly low since ICMEs lead to magnetic storms showing a minimum of SYM-H below $-60 \mathrm{nT}$ in more than $70 \%$ of the events (Echer et al., 2005). There are two possibilities for explaining such a low amplitude. The event list could involve some very ineffective events reducing the statistical average. However, the average of the minimums of SYM-H in our list is equal to $-72.4 \mathrm{nT}$. The second, more likely possibility is that the maximal amplitude of the magnetic storm can occur at different times during the event crossing. This would lead to a flattening of the average and would explain such low amplitudes.

First results obtained here show the average characteristics of an event related to an ICME. It is composed of two substructures (sheath and ICME), both of them being possibly geoeffective and impacting all the current systems. The former abruptly impact the magnetosphere, displaying a strong 
increase in all solar wind parameters and the magnetosphere is still disturbed when the latter one arrives. This means that the two substructures cannot be studied separately and the event (sheath + ICME) must be seen as a whole since the two substructures are deeply linked. Therefore, the global reaction of the magnetosphere is the sum of the contribution of each substructure. Secondly, the range of possible reactions of the magnetosphere is very large in terms of geoeffectiveness (maximal amplitudes of the indices) but also in terms of dynamics of the magnetic storm (when does this maximum occur). Thereafter, we sort the events according to large-scale features (the presence of a shock upstream of the ICME and the magnetic configuration of the ICME).

\section{Presence of a shock and magnetic structure}

\subsection{Results}

Figure 3 displays SEAs of the same three solar wind parameters and two geomagnetic indices as in Fig. 2. The 306 events are sorted into four groups according to the presence of a shock and the magnetic configuration (MC or ejecta). The four groups are ejecta without shock (green line), MCs without shock (orange), ejecta with shocks (blue), and MCs with shocks (red). They involve, respectively, 87, 17, 150, and 52 events.

First, the profiles of the parameters during the sheath are highly dependent on the presence of a shock. The abrupt increases observed in Fig. 2 are essentially due to the shockassociated events (in red and blue), which display a massive and abrupt increase of all parameters at the first epoch time. For events without shock, the pressure also increases abruptly but to lower values, and the magnetic field clearly increases gradually. In addition, the pressure and the magnetic field increase a bit more strongly for MCs than for ejecta (orange with respect to green line and red with respect to blue line). During the ICME itself, the pressure falls down quickly to low values and hence shows no significant differences between the groups. Conversely, we observe four distinct magnetic field profiles that depend on the two features. Ejecta without shock (in green) display a low amplitude of magnetic field during the overall event. If ejecta are associated with shock (in blue), the magnetic field amplitude is increased during the sheath and the first part of the ICME. During the second part of the ICME, it recovers low values similar to those of ejecta without shock. Although the magnetic field amplitude of MCs without shock (in orange) is quite low during the sheaths, it strongly increases within the ICMEs, being maximal near their centers. In the case of MC with shock (in red), the magnetic field has high values during the sheath and the ICME. In the second half of the ICME, the values are similar to those of MCs without shock.

SEAs of speed show that shock-associated events have higher speed during the overall event, which is expected
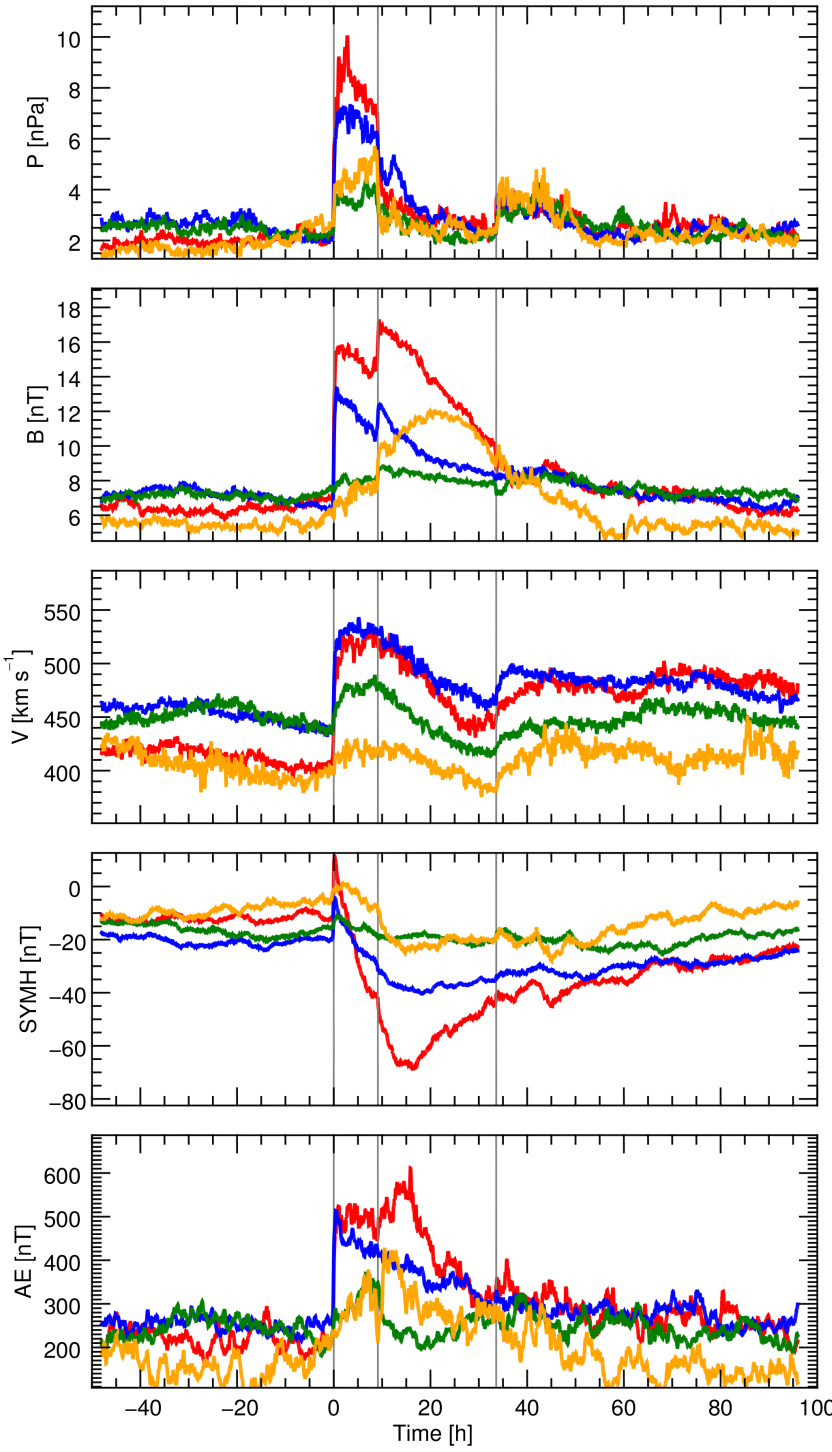

Figure 3. SEAs of three $\mathrm{SW}$ parameters $(B, P, V)$ and two geomagnetic indices (SYM-H and AE) for 306 ICMEs between 1996 and 2008. The three vertical lines are the epoch times indicating (respectively from left to right) the onset of the sheath, the onset of the ICME, and the end of the ICME. The different colors correspond to different subsets of events: ejecta without shock (87 events) are displayed in green, MC without shock (17) in orange, ejecta with shock (150) in blue, and MC with shock in red (52).

since the presence of a shock depends, by definition, on the speed of the event. However, we notice the unexpectedly low speed of MCs without shock (in orange).

The groups also display some differences in solar wind parameters before the event onset. All displayed solar wind parameters are significantly lower for MCs (orange and red) than for ejecta (green and blue), in particular the speed. 
The two bottom panels in Fig. 3 give SEAs of two geomagnetic indices (SYM-H and AE). The four groups cause distinct reactions in the magnetosphere.

During the sheath, if there is a shock, SYM-H reacts, first, by a positive peak at the first epoch time due to an increase in the magnetopause current and then by a decrease during the overall sheath. If there is no shock, then SYM-H barely reacts during the sheath and the values at the first and second epoch times are almost the same. During the ICME, two processes must be taken into account. The strong decrease in the pressure at the second epoch time causes a weakening of the magnetopause current, which leads to a decrease in SYM-H. In addition, the intensification of the ring current also induces a decrease in SYM-H. Since the two processes obviously occur during the first hours of the ICME, it is difficult to estimate their respective contributions. However, it is shown that MCs (with shock in red and without shock in orange) cause the strongest decreases in SYMH. Variations in SYM-H during ejecta without shock (in green) are very weak and those of ejecta with shock are intermediate. Observations of SEAs of AE bring complementary information. Again, the wall structure is observed at the first epoch time for shock-associated events and intensification of auroral electrojets remains high during the sheath. For events without shock, despite the fact that SYM-H does not react, there is an increase in AE. Even though this increase is weak and progressive (reminding us of the variations in $B$ ), it does exist and is not negligible. During the ICMEs, AE increases in the first part of the MCs (red and orange) and then decreases in the second part, while it decreases during the overall ejecta (green and blue).

\subsection{Discussion}

The results reveal the effects of these features (presence of a shock, magnetic structure) on the profiles of solar wind parameters. During the sheath, it has been shown that the amplitude of all solar wind parameters strongly depends on the presence of a shock. However, it has been noticed that they also slightly depend on the magnetic structure (ejecta or $\mathrm{MC})$. The pressure and the magnetic field increase, on average, more strongly for $\mathrm{MC}$ with shock (in red) than for ejecta with shock (in blue) and for MC without shock (in orange) than for ejecta without shock (in green). We believe that this difference is related to the pre-event solar wind conditions, which are not the same for MCs and ejecta. Indeed, we showed that all solar wind parameters (in particular the speed) of the upstream solar wind are lower for MCs than ejecta. As a consequence, even if the speeds during the events are similar, the rate of change of speed at the first epoch time is higher upstream of MCs than upstream of ejecta, which may account for the stronger magnetic field during the sheath. However, the explanation for this difference in initial conditions is not clear, but it is important to note that they are different for the two subsets of ICMEs (ejecta and MC) and
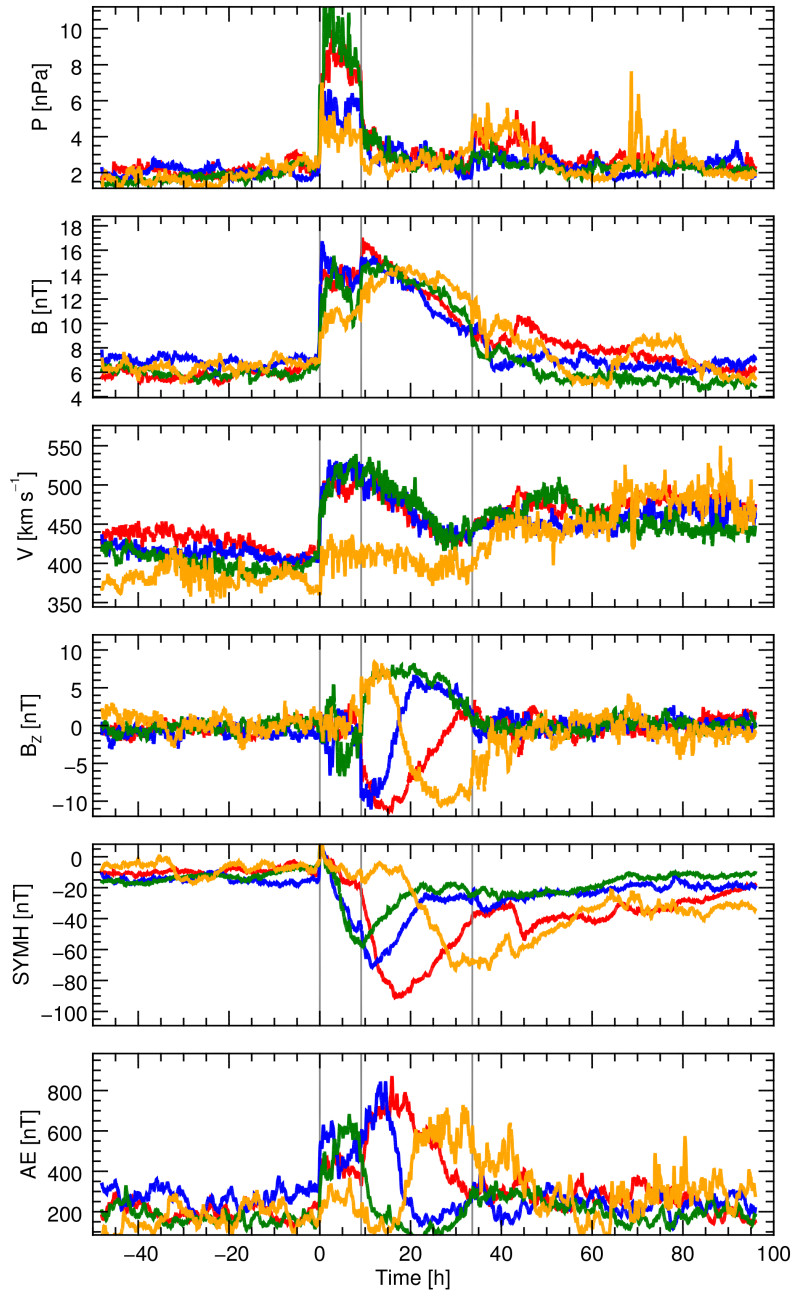

Figure 4. SEAs of four $\mathrm{SW}$ parameters $\left(B, P, V, B_{Z}\right)$ and two geomagnetic indices (SYM-H and AE) for 69 magnetic clouds between 1996 and 2008. The three vertical lines are the epoch times indicating (respectively from left to right) the onset of the sheath, the onset of the ICME, and the end of the ICME. The different colors correspond to different polarities of magnetic clouds: full north (17 events) in green, north-south (9) in orange, south-north (16) in blue, and full south (27) in red.

this implies some differences in solar wind parameter amplitude during the sheath.

The shock causes a strong reaction of the overall current system. If there is no shock, SYM-H barely reacts and AE increases progressively and weakly. This provides crucial indication of the dissipation of energy into the different currents of the magnetosphere. During the sheath crossing, if there is no shock, then only a modest quantity of energy enters the magnetosphere. In this case, the energy seems to move preferably toward the electrojets, which are sufficient enough to dissipate that energy. Thus, the ring current is not involved. These results are in good agreement with the results of Huttunen and Koskinen (2004), who showed that sheaths, with 
highly oscillating $B_{Z}$, are more likely to make $\mathrm{AE}$ react than SYM-H. Conversely, if there is a shock, a huge amount of energy abruptly enters the entire magnetosphere, including the ring current.

Recently, Yermolaev et al. (2015) investigated several large-scale types of solar wind. They proposed a description of a few solar wind parameters and magnetic indices during ICMEs using a method similar to ours. Our results and interpretations confirm and complete those of Yermolaev et al. (2015), notably about the magnetic field amplitude during ejecta and $\mathrm{MC}$ with or without shock. The magnetic field amplitude during the ICME depends on both features. MCs display a higher magnetic field than ejecta and their amplitude is the highest near the center of the MC. However, the presence of a shock tends to increase the magnetic field during the first part of the ICME. Thus, MCs without shocks (in orange) display a magnetic field amplitude that is consistent with the Lundquist solution for MCs (Lundquist, 1950; Burlaga, 1988). If the MC is preceded by a shock, then the sheath compresses the MC, increasing the magnetic field in its first half. First, this explains why it is higher for ejecta with shock (in blue) than for ejecta without shock (in green), and secondly, this explains why MCs with shock (in red) have higher magnetic field amplitude than MCs without shock (in orange) during the first part of the magnetic cloud. These distinctions in the solar wind parameters also seem to have a direct impact on the reaction of geomagnetic indices, especially for MCs with shock, which logically cause the strongest reaction. The second strongest reaction during the ICMEs is caused by MC without shock, confirming that MCs are more effective in disturbing the ring current than ejecta. However, one can note the decrease in SYMH in the first part of the ejecta associated with shock (in blue). This is related to the presence of a shock, causing an increase in the magnetic field amplitude at this time.

\section{Polarity of magnetic clouds}

\subsection{Results}

To further investigate the geoeffectiveness of the 69 magnetic clouds, we divide them into four groups according to their polarity. The polarity of a magnetic cloud is defined with respect to the sign of the $z$ component of the magnetic field $\left(B_{Z}\right)$. MCs are therefore sorted as follows: fullnorth (FN-MC, 17 events) in green, north-south (NS-MC, 9 events) in orange, south-north (SN-MC, 16 events) in blue, and full-south (FS-MC, 27 events) in red. The higher number of events in the SN group (16) over those in the NS group (9) is consistent with the study of Mulligan et al. (1998), suggesting that the number of SN polarity prevails the NS polarity during the odd solar cycle. However, we underline that the number of events for each group is considerably reduced, and thus statistical results should be considered care-

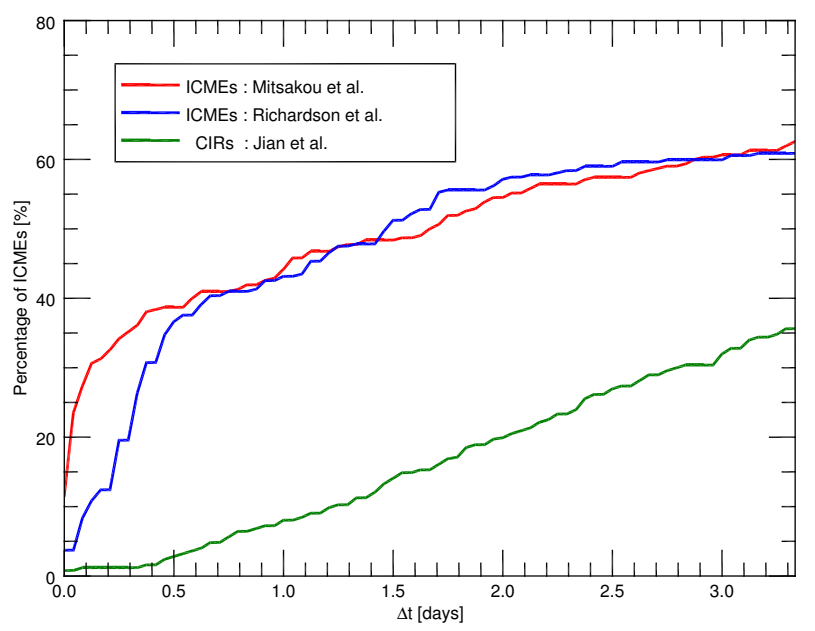

Figure 5. Percentage of events involved in a sequence according to $\Delta t$. Two events are involved in a sequence if they are separated by less than $\Delta t$ days. For comparison purpose, the red and blue lines give the results for two lists: Mitsakou and Moussas (2014b) (in red) and Cane and Richardson (2003) (in blue). We also added the results for a list of CIRs from Jian et al. (2006) study (in green).

fully. Figure 4 gives SEAs of four solar wind parameters $(P$, $B, V$, and $B_{Z}$ ) and two geomagnetic indices (SYM-H and $\mathrm{AE})$.

All groups display some relatively similar profiles of pressure, magnetic field amplitude, and speed despite two significant differences. First, the speed of NS-MCs are particularly low and do not display the typical and expected variations (strong increase at the first epoch time and slow decrease during the ICME). Secondly, the pressure during the sheath is lower for bipolar MCs (orange and blue) compared to unipolar (blue and red) ones. Apart from this, the only meaningful difference is observed for $B_{Z}$ since the four groups display four strong and distinct rotations of their magnetic field.

SEAs of geomagnetic indices indicate that the different groups cause very specific profiles of magnetic storms. FSMCs cause the strongest reaction and SYM-H reaches its maximal amplitude near the center of the cloud. Comparing the SEA of SYM-H for SN-MCs and NS-MCs, one can observe that the minimal values are similar despite two very distinct temporal profiles. For SN-MCs, SYM-H continues the decrease started during the sheath and reaches its minimal value in the first half of the cloud. Conversely, for NS-MCs, SYM-H recovers from the disturbances due to the sheath during the first half of the cloud and then decreases again during the second half, forming a two-peak magnetic storm. For FN-MCs, the maximal amplitude of SYM-H is reached at the second epoch time and SYM-H recovers all along the magnetic cloud. 


\subsection{Discussion}

In addition to confirming some well-known results (e.g., FSMCs are the most geoeffective; see e.g., Huttunen et al. (2005)), our approach leads to original results concerning the dynamics of the magnetic storms. SN-MCs and NSMCs have similar geoefficiencies, which confirm the results obtained by $\mathrm{Li}$ and Luhmann (2004) or by Kilpua et al. (2012), who compare the geoeffectiveness of SN-MCs and NS-MCs using the Burton et al. (1975) Dst formula. However, they display very different temporal profiles since the disturbances due to the magnetic clouds could either come in addition to those of the sheaths (for SN-MCs) or form twopeak storms (for NS-MCs). A previous study by Fenrich and Luhmann (1998) pointed out that approximately $40 \%$ of the magnetic clouds are followed by a fast solar wind, which can cause an increase in the magnetic field amplitude toward the latter part of the magnetic cloud most of the time. Therefore, they argued that in the case of NS-MCs, the increase in magnetic field amplitude when the interplanetary magnetic field $B_{Z}$ is negative could increase their geoeffectiveness. The apparent opposite results of Fenrich and Luhmann (1998) to those of Li and Luhmann (2004) and Kilpua et al. (2012) could therefore be explained by our results. On the first hand, the geoeffectiveness of NS-MCs is increased by the following high-speed solar wind. On the other hand, the geoeffectiveness of SN-MCs is increased by the contribution of the preceding sheath (also observed by Echer and Gonzalez (2004)). All in all, the average intensities of the magnetic storms are similar for the two subsets of MCs and our panel of events.

We recall that these results should be taken with caution because of the small number of events (16 and 9). It is interesting to note that NS-MCs are geoeffective in the second half of the MCs despite their unusually low speed. This suggests that speed does not play a crucial role in the geoeffectiveness of MCs. This is supported by Tsurutani et al. (2004), who studied the properties and geoeffectiveness of slow magnetic clouds. They found that slow magnetic clouds were surprisingly geoeffective due to large electric fields, despite their low speed. It is also clear that FN-MCs are not geoeffective and that the magnetic storms caused during their crossing are exclusively attributed to the sheaths preceding them. The polarity of magnetic clouds is a crucial feature since it determines not only their geoeffectiveness but also the time profile of the magnetic storms. Thus, depending on the polarity of the $\mathrm{MC}$, the maximal amplitude of the storm can be reached either near the onset of the MC (for FN-MCs) or at its end (for NS-MCs), i.e., with more than $24 \mathrm{~h}$ between them.
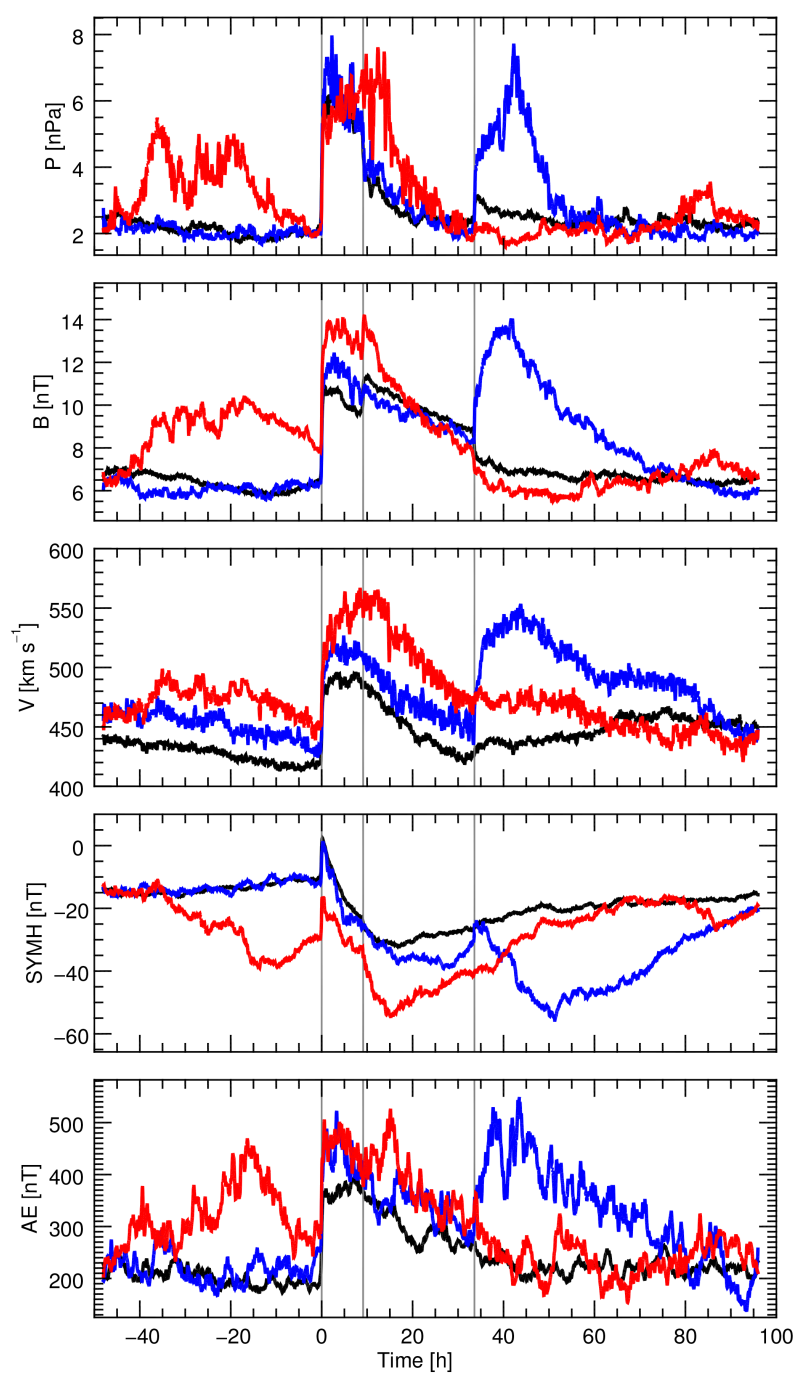

Figure 6. SEAs of three SW parameters $(B, P, V)$ and two geomagnetic indices (SYM-H and AE) for 306 ICMEs between 1996 and 2008. The three vertical lines are the epoch times indicating (respectively from left to right) the onset of the sheath, the onset of the ICME, and the end of the ICME. The different colors correspond to different subset of events: isolated events (black line, 187 events), first events (in blue, 34 events), and second events (in red, 34 events).

\section{Sequences of events}

\subsection{Results}

Here, we aim to focus on the sequences of ICMEs using the same SEA method as in previous sections. We define the sequence of events as follows. Two ICMEs are considered as involved in a sequence if they follow one another quickly, i.e., if the time interval between them (between the end of the first ICME and the sheath onset of the second one) is less than a given time $\Delta t$. In Fig. 5, we plot the percentage of ICMEs involved in a sequence as a function of $\Delta t$. In or- 
der to validate the observations, we use two lists of ICMEs from two different studies: Mitsakou and Moussas (2014b) (on which our list is based) and Cane and Richardson (2003). For comparison purposes, we add a similar plot for a list of co-rotating interaction regions (CIRs) (Jian et al. (2006), sequences of CIRs being defined similarly). The two lists of ICMEs indicate that ICMEs have a strong tendency to follow one another and to form sequences of events (which is not true for CIRs). Indeed, if we set $\Delta t=0.5$ days, $40 \%$ of ICMEs are involved in a sequence. As can be seen in Figure 2, geomagnetic indices (especially SYM-H) are not completely recovered $12 \mathrm{~h}$ after the end of the ICMEs. This means that for a non-negligible number of events, they impact a magnetosphere that is already influenced by a previous event. In previous sections, events were considered as isolated structures and initial state of the magnetosphere was not been taken into account. In this section, we focus on the effect of such sequences of events.

We set $\Delta t=0.5$ days. All in all, 187 events are found to be isolated and 119 are involved in a sequence. Among them, we found 34 sequences of two events, 10 sequences of three events, 4 sequences of four events, and 1 sequence of five events. In this section, we keep only isolated events and those involved in a sequence of two. Three subsets are considered: isolated (187 events), first of a sequence (34, hereafter refereed to as "first events"), and second of a sequence (34, "second events").

In Fig. 6, we plot SEAs for the same parameters as previous sections for the three considered groups. Isolated events are displayed in black, first events in blue, and second events in red. As expected, the three lines display strong differences before and after the event crossings. Isolated events display low solar wind parameters before and after the event crossing. Conversely, we can easily see the presence of other events, either following the first events of the sequences (in blue) or preceding the second events (in red). Solar wind parameters during the events are relatively similar for all groups. The pressures during the sheath are similar, while the peak of pressure in the first part of the ICME for second events (in red) might be due to the effect of one singular event. The magnetic field during the sheath is also slightly higher for the second events, probably due to the corresponding higher speed.

The two bottom panels of Fig. 6 show SEAs of geomagnetic indices AE and SYM-H for the same three groups. The black line is the SEA for isolated events and shows the typical profile of a magnetic storm that is not disturbed by other events. The blue line corresponds to the first events of the sequences and both indices are relatively close to those of isolated events. The main differences occur for second events (red line), especially for SYM-H. In these cases, SYM-H displays higher amplitude at the first epoch time. Then, during the event, SYM-H displays the expected decrease in SYM$\mathrm{H}$, which reaches amplitudes much higher than for isolated or first events.

\subsection{Discussion}

These results point out the importance of considering the initial state of the magnetosphere. First, it has been shown that sequences of ICMEs are very common. Out of our list, $40 \%$ of the ICMEs were involved in a sequence. This reveals some characteristics of solar wind parameters seen in Fig. 2. The relatively high values of pressure, magnetic field, and speed after the third epoch time is clearly credited to the presence of other events in a non-negligible number of cases. Belonging to a sequence does not seem to strongly affect the profile of the solar wind parameters during the event crossing itself (between the first and the third epoch time). They are similar, except for the higher speed of second events, which can be seen as a cause of the sequence. Indeed, if two ICMEs escape the sun and the second one is faster than the first one, then it can catch it up and form a sequence. In the opposite case, the first event will outrun the second one and there will be no sequence. Despite relatively similar solar wind parameters, the profile of geomagnetic indices is strongly different since SYM-H reaches much higher amplitude for the second events of sequences. These results directly confirm those of Eselevich and Fainshtein (1993) since we showed that in the case of a sequence of events, the amplitude of the second magnetic storm (in terms of SYM-H) is statistically higher than that of the first one. The observation of the temporal profile brings additional information. The relative decrease in SYM-H during the crossing of the second event (difference between SYM-H at the first epoch time and the minimal value of SYM-H) is close to that of the first event ( $\triangle \mathrm{SYM}-\mathrm{H}$ $\approx-25 n T)$. The difference found in the magnetic storm intensity is thus related to the initial state of the magnetosphere being much more disturbed when the second events impact it.

We underline that these are statistical results and that for some specific cases, the preconditioning could not play a role in the development of the magnetic storm (see e.g., Kozyra et al. (2002)).

\section{Summary}

We performed a statistical analysis of 306 ICMEs and their induced magnetic storms over the period 1996-2008. While most of studies using SEA consider the minimum value of Dst as the epoch time, we chose three epoch times related to the structure of the ICMEs (following a framework similar to those of Yermolaev et al. (2015) and Kilpua et al. (2015)). Then we sorted events according to large-scale features (presence of a shock, magnetic structure, polarity, belonging to a sequence). On the one hand, our study reproduces some well-known results and, on the other hand, it shows new elements that reveal the variability of the dynamics of magnetic storms caused by ICMEs. 
First, our study shows the necessity of considering each event (sheath + ICME) as a whole. Indeed, the global magnetic storm is related to both contributions of the sheath and the ICME and most of the time the magnetosphere is still undergoing the disturbances from the sheath when the ICME impacts it.

By sorting the events according to large-scale features, we revealed the main characteristics of geoeffective events, supporting and completing previous studies (e.g., Yermolaev et al., 2015). The geoeffectiveness of the sheath is strongly related to the presence of a shock. The geoeffectiveness of the ICME is related to both features. The compression between the sheath and the ICME causes an increase in the magnetic field amplitude during the first part of the ICME, which might increase its geoeffectiveness. The faster the ICME propagates, the stronger this increase is. Thus, this effect is essentially observed for shock-associated events. Concerning the magnetic structures, MCs cause stronger magnetic disturbances than ejecta, which barely makes SYM-H react most of the time. Our results also confirm these of Huttunen and Koskinen (2004), who found that the sheaths disturb the electrojets more than the ring current.

It is also interesting to note that ambient solar wind conditions are not the same for MCs and ejecta. This is an important result since it suggests that the initial state of the magnetosphere is more relaxed before a MC-related event.

To our knowledge, it is the first time the effects of the polarity of magnetic clouds are studied using SEA. Our approach provides a better understanding of the variability of magnetic storms caused by MCs. The polarity impacts not only the intensity of the magnetic storms but also their temporal profile. In the case of SN-MCs, the contribution of the MC comes in addition to that of the sheath, in contrast to NS-MCs, where the sheath and the magnetic cloud trend to form a two-step storm. The geoeffectiveness of SN-MCs is increased by the contribution of the preceding sheath, while that of NS-MCs could be increased by the following highspeed solar wind (Fenrich and Luhmann, 1998). This could explain why there is no clear difference in geoeffectiveness for these two subsets of MCs (agreeing with the previous study of Li and Luhmann (2004)).

Full-south magnetic clouds following shocks are, on average, clearly the most geoeffective structures since each substructure (sheath following a shock and FS-MCs) strongly disturbs the magnetosphere. Conversely, ejecta or FN-MCs, if they are not fast enough to induce the development of a shock upstream from them, will cause only modest disturbances, more likely in the auroral electrojets.

ICMEs have a strong tendency to form sequences of events following one another. Between 1996 and 2008, we found that $40 \%$ of the ICMEs belong to such sequences. Our results support the results of other studies that show that these sequences are an important source of geoeffectiveness (e.g., Eselevich and Fainshtein, 1993; Echer and Gonzalez, 2004; Skoug et al., 2004; Farrugia et al., 2006). However, we showed that although second events usually propagate faster than the first ones, these events do not seem to affect each other and their structures are roughly similar to those of isolated events. This result suggests that the stronger reaction of the magnetosphere is clearly due to the sequence itself. Thus, this underlines the relevance of the initial state of the magnetosphere when an event impacts it. It could also put into question the notion of "extreme events" since several modest events could produce as much magnetic disturbance as one strong event.

\section{Data availability}

The data are publicly accessible. They can be found at the websites http://isgi.unistra.fr and http://omniweb.gsfc.nasa. gov.

\section{The Supplement related to this article is available online at doi:10.5194/angeo-35-147-2017-supplement.}

Competing interests. The authors declare that they have no conflict of interest.

Acknowledgements. Remi Benacquista's thesis is co-financed by ONERA and CNES. The results presented in this paper rely on geomagnetic indices calculated and made available by ISGI Collaborating Institutes from data collected at magnetic observatories. We thank the involved national institutes, the INTERMAGNET network, and ISGI (isgi.unistra.fr). The authors are grateful to the OMNI database for the use of the data, obtained at http://omniweb.gsfc.nasa.gov.

The topical editor, G. Balasis, thanks the two anonymous referees for help in evaluating this paper.

\section{References}

Akasofu, S.-I.: Energy coupling between the solar wind and the magnetosphere, Space Sci. Rev., 28, 121-190, 1981.

Borovsky, J. E. and Denton, M. H.: Magnetic field at geosynchronous orbit during high-speed stream-driven storms: Connections to the solar wind, the plasma sheet, and the outer electron radiation belt, J. Geophys. Res., 115, A08217, doi:10.1029/2009JA015116, 2010.

Burlaga, L.: Magnetic Clouds and Force-Free Fields With Constant Alpha, J. Geophys. Res., 93, 7217-7224, 1988.

Burlaga, L. F.: Terminology for ejecta in the solar wind, EOS, 82, 433-433, doi:10.1029/01EO00265, 2001.

Burlaga, L., Sittler, E., Mariani, F., and Schwenn, R.: Magnetic loop behind an interplanetary shock: Voyager, Helios, and IMP 8 observations, J. Geophys. Res., 86, 6673-6684, doi:10.1029/JA086iA08p06673, 1981. 
Burton, R. K., McPherron, R. L., and Russell, C. T.: An empirical relationship between interplanetary conditions and Dst, J. Geophys. Res., 80, 4204-4214, doi:10.1029/JA080i031p04204, 1975.

Cane, H. V. and Richardson, I. G.: Interplanetary coronal mass ejections in the near-Earth solar wind during 1996-2002, J. Geophys. Res., 108, 1156, doi:10.1029/2002JA009817, 2003.

Crooker, N.: Solar and heliospheric geoeffective disturbances, J. Atmos. Sol.-Terr. Phy., 62, 1071-1085, doi:10.1016/S13646826(00)00098-5, 2000.

Denton, M. H., Borovsky, J. E., Skoug, R. M., Thomsen, M. F., Lavraud, B., Henderson, M. G., McPherron, R. L., Zhang, J. C., and Liemohn, M. W.: Geomagnetic storms driven by ICMEand CIR-dominated solar wind, J. Geophys. Res., 111, A07S07, doi:10.1029/2005JA011436, 2006.

Dumbović, M., Devos, A., Vršnak, B., Sudar, D., Rodriguez, L., Ruždjak, D., Leer, K., Vennerstrøm, S., and Veronig, A.: Geoeffectiveness of Coronal Mass Ejections in the SOHO Era, Sol. Phys., 290, 579-612, doi:10.1007/s11207-014-0613-8, 2015.

Echer, E. and Gonzalez, W. D.: Geoeffectiveness of interplanetary shocks, magnetic clouds, sector boundary crossings and their combined occurrence: GEOEFFECTIVENESS OF IP STRUCTURES, Geophys. Res. Lett., 31, L09808, doi:10.1029/2003GL019199, 2004.

Echer, E., Alves, M., and Gonzalez, W.: A statistical study of magnetic cloud parameters and geoeffectiveness, J. Atmos. Sol.-Terr. Phys., 67, 839-852, doi:10.1016/j.jastp.2005.02.010, 2005.

Eselevich, V. and Fainshtein, V. G.: An investigation of the relationship between the magnetic storm Dst-index and different types of solar wind streams, Ann. Geophys., 11, 678-684, 1993.

Farrugia, C., Matsui, H., Kucharek, H., Jordanova, V., Torbert, R., Ogilvie, K., Berdichevsky, D., Smith, C., and Skoug, R.: Survey of intense Sun-Earth connection events (1995-2003), Adv. Space Res., 38, 498-502, doi:10.1016/j.asr.2005.05.051, 2006.

Fenrich, F. R. and Luhmann, J. G.: Geomagnetic response to magnetic clouds of different polarity, Geophys. Res. Lett., 25, 29993002, doi:10.1029/98GL51180, 1998.

Ganushkina, N. Y., Liemohn, M. W., Dubyagin, S., Daglis, I. A., Dandouras, I., De Zeeuw, D. L., Ebihara, Y., Ilie, R., Katus, R., Kubyshkina, M., Milan, S. E., Ohtani, S., Ostgaard, N., Reistad, J. P., Tenfjord, P., Toffoletto, F., Zaharia, S., and Amariutei, O.: Defining and resolving current systems in geospace, Ann. Geophys., 33, 1369-1402, doi:10.5194/angeo-33-1369-2015, 2015.

Gonzalez, W. D., de Gonzalez, A. L. C., Dal Lago, A., Tsurutani, B. T., Arballo, J. K., Lakhina, G. K., Buti, B., Ho, C. M., and $\mathrm{Wu}$, S.-T.: Magnetic cloud field intensities and solar wind velocities, Geophys. Res. Lett., 25, 963-966, doi:10.1029/98GL00703, 1998.

Gopalswamy, N.: Solar connections of geoeffective magnetic structures, J. Atmos. Sol.-Terr. Phys., 70, 2078-2100, doi:10.1016/j.jastp.2008.06.010, 2008.

Gosling, J. T., Russell, C. T., Priest, E. R., and Lee, L. C.: Coronal mass ejections and magnetic flux ropes in interplanetary space, in: Geophysical Monograph Series, American Geophysical Union, Washington, DC, 58, 343-364, http://doi.wiley.com/ 10.1029/GM058p0343, 1990.

Guo, J., Feng, X., Emery, B. A., Zhang, J., Xiang, C., Shen, F., and Song, W.: Energy transfer during intense geomagnetic storms driven by interplanetary coronal mass ejections and their sheath regions: ENERGY TRANSFER, J. Geophys. Res., 116, A05106, doi:10.1029/2011JA016490, 2011.

Hietala, H., Kilpua, E. K. J., Turner, D. L., and Angelopoulos, V.: Depleting effects of ICME-driven sheath regions on the outer electron radiation belt, Geophys. Res. Lett., 41, 2258-2265, doi:10.1002/2014GL059551, 2014.

Huttunen, K. E. J. and Koskinen, H. E. J.: Importance of post-shock streams and sheath region as drivers of intense magnetospheric storms and high-latitude activity, Ann. Geophys., 22, 1729-1738, doi:10.5194/angeo-22-1729-2004, 2004.

Huttunen, K. E. J., Huttunen, J., Koskinen, H., and Schwenn, R.: Variability of magnetospheric storms driven by different solar wind perturbations, J. Geophys. Res., 107, doi:10.1029/2001JA900171, 2002.

Huttunen, K. E. J., Schwenn, R., Bothmer, V., and Koskinen, H. E. J.: Properties and geoeffectiveness of magnetic clouds in the rising, maximum and early declining phases of solar cycle 23, Ann. Geophys., 23, 625-641, doi:10.5194/angeo-23-625-2005, 2005.

Ilie, R., Liemohn, M. W., Thomsen, M. F., Borovsky, J. E., and Zhang, J.: Influence of epoch time selection on the results of superposed epoch analysis using ACE and MPA data, J. Geophys. Res., 113, A00A14, doi:10.1029/2008JA013241, 2008.

Jian, L., Russell, C. T., Luhmann, J. G., and Skoug, R. M.: Properties of Stream Interactions at One AU During 1995-2004, Sol. Phys., 239, 337-392, doi:10.1007/s11207-006-0132-3, 2006.

Kilpua, E., Jian, L., Li, Y., Luhmann, J., and Russell, C.: Multipoint ICME encounters: Pre-STEREO and STEREO observations, J. Atmos. Sol.-Terr. Phys., 73, 1228-1241, doi:10.1016/j.jastp.2010.10.012, 2011.

Kilpua, E. K. J., Li, Y., Luhmann, J. G., Jian, L. K., and Russell, C. T.: On the relationship between magnetic cloud field polarity and geoeffectiveness, Ann. Geophys., 30, 1037-1050, doi:10.5194/angeo-30-1037-2012, 2012.

Kilpua, E. K. J., Isavnin, A., Vourlidas, A., Koskinen, H. E. J., and Rodriguez, L.: On the relationship between interplanetary coronal mass ejections and magnetic clouds, Ann. Geophys., 31, 1251-1265, doi:10.5194/angeo-31-1251-2013, 2013.

Kilpua, E. K. J., Hietala, H., Turner, D. L., Koskinen, H. E. J., Pulkkinen, T. I., Rodriguez, J. V., Reeves, G. D., Claudepierre, S. G., and Spence, H. E.: Unraveling the drivers of the storm time radiation belt response: RADIATION BELT AND STORM DRIVERS, Geophys. Res. Lett., 42, 3076-3084, doi:10.1002/2015GL063542, 2015.

King, J. H. and Papitashvili, N.: Solar wind spatial scales in and comparisons of hourly Wind and ACE plasma and magnetic field data, J. Geophys. Res., 110 A02104, doi:10.1029/2004JA010649, 2005.

Kozyra, J. U., Liemohn, M. W., Clauer, C. R., Ridley, A. J., Thomsen, M. F., Borovsky, J. E., Roeder, J. L., Jordanova, V. K., and Gonzalez, W. D.: Multistep Dst development and ring current composition changes during the 4-6 June 1991 magnetic storm: multistep DST and ring current composition, J. Geophys. Res., 107, 1-2, doi:10.1029/2001JA000023, 2002.

Lavraud, B., Thomsen, M. F., Borovsky, J. E., Denton, M. H., and Pulkkinen, T. I.: Magnetosphere preconditioning under northward IMF: Evidence from the study of coronal mass ejection and corotating interaction region geoeffectiveness, J. Geophys. Res., 111, A09208, doi:10.1029/2005JA011566, 2006. 
Lepping, R. P., Wu, C.-C., and Berdichevsky, D. B.: Automatic identification of magnetic clouds and cloud-like regions at $1 \mathrm{AU}$ : occurrence rate and other properties, Ann. Geophys., 23, 26872704, doi:10.5194/angeo-23-2687-2005, 2005.

Lepping, R. P., Berdichevsky, D. B., Wu, C.-C., Szabo, A., Narock, T., Mariani, F., Lazarus, A. J., and Quivers, A. J.: A summary of WIND magnetic clouds for years 1995-2003: model-fitted parameters, associated errors and classifications, Ann. Geophys., 24, 215-245, doi:10.5194/angeo-24-215-2006, 2006.

Li, Y. and Luhmann, J.: Solar cycle control of the magnetic cloud polarity and the geoeffectiveness, J. Atmos. Sol.-Terr. Phys., 66, 323-331, doi:10.1016/j.jastp.2003.12.001, 2004.

Liu, Y. D., Luhmann, J. G., Kajdič, P., Kilpua, E. K., Lugaz, N., Nitta, N. V., Möstl, C., Lavraud, B., Bale, S. D., Farrugia, C. J., and Galvin, A. B.: Observations of an extreme storm in interplanetary space caused by successive coronal mass ejections, Nat. Commun., 5, 3481, doi:10.1038/ncomms4481, 2014.

Lundquist, S.: Ark. Fys., 361-365, 1950.

Lyatsky, W. and Tan, A.: Solar wind disturbances responsible for geomagnetic storms, J. Geophys. Res., 108, 1134, doi:10.1029/2001JA005057, 2003.

Menvielle, M., Iyemori, T., Marchaudon, A., and Nosé, M.: Geomagnetic Indices, in: Geomagnetic Observations and Models, edited by: Mandea, M. and Korte, M., Springer Netherlands, Dordrecht, 183-228, 2011.

Mitsakou, E. and Moussas, X.: Erratum to: Statistical Study of ICMEs and Their Sheaths During Solar Cycle 23 (1996-2008), Sol. Phys., 289, 4413-4421, doi:10.1007/s11207-014-0575-x, 2014a.

Mitsakou, E. and Moussas, X.: Statistical Study of ICMEs and Their Sheaths During Solar Cycle 23 (1996-2008), Sol. Phys., 289, 3137-3157, doi:10.1007/s11207-014-0505-y, 2014b.

Miyoshi, Y. and Kataoka, R.: Ring current ions and radiation belt electrons during geomagnetic storms driven by coronal mass ejections and corotating interaction regions, Geophys. Res. Lett., 32, L21105, doi:10.1029/2005GL024590, 2005.

Mulligan, T., Russell, C. T., and Luhmann, J. G.: Solar cycle evolution of the structure of magnetic clouds in the inner heliosphere, Geophys. Res. Lett., 25, 2959-2962, doi:10.1029/98GL01302, 1998.

Owens, M. J. and Cargill, P.: Correlation of magnetic field intensities and solar wind speeds of events observed by ACE, J. Geophys. Res., 107, doi:10.1029/2001JA000238, 2002.

Pulkkinen, T., Palmroth, M., Tanskanen, E., Ganushkina, N., Shukhtina, M., and Dmitrieva, N.: Solar wind - magnetosphere coupling: A review of recent results, J. Atmos. Sol.-Terr. Phys., 69, 256-264, doi:10.1016/j.jastp.2006.05.029, 2007.

Richardson, I. G. and Cane, H.: The fraction of interplanetary coronal mass ejections that are magnetic clouds: Evidence for a solar cycle variation, Geophys. Res. Lett., 31, L18804, doi:10.1029/2004GL020958, 2004.

Richardson, I. G. and Cane, H. V.: Solar wind drivers of geomagnetic storms during more than four solar cycles, Journal of Space Weather and Space Climate, 2, A01, A09102, doi:10.1051/swsc/2012001, 2012.

Skoug, R. M., Gosling, J., Steinberg, J., and McComas, D.: Extremely high speed solar wind: 29-30 October 2003, J. Geophys. Res., 109, 8519-8531, doi:10.1029/2004JA010494, 2004.
Tsurutani, B. T., Gonzalez, W. D., Tang, F., Akasofu, S. I., and Smith, E. J.: Origin of interplanetary southward magnetic fields responsible for major magnetic storms near solar maximum (1978-1979), J. Geophys. Res., 93, 8519-8531, doi:10.1029/JA093iA08p08519, 1988.

Tsurutani, B., Gonzalez, W., Zhou, X.-Y., Lepping, R., and Bothmer, V.: Properties of slow magnetic clouds, J. Atmos. Sol.-Terr. Phys., 66, 147-151, doi:10.1016/j.jastp.2003.09.007, 2004.

Turner, N. E., Cramer, W. D., Earles, S. K., and Emery, B. A.: Geoefficiency and energy partitioning in CIR-driven and CMEdriven storms, J. Atmos. Sol.-Terr. Phys., 71, 1023-1031, doi:10.1016/j.jastp.2009.02.005, 2009.

Veselovsky, I. S., Panasyuk, M. I., Avdyushin, S. I., Bazilevskaya, G. A., Belov, A. V., Bogachev, S. A., Bogod, V. M., Bogomolov, A. V., Bothmer, V., Boyarchuk, K. A., Vashenyuk, E. V., Vlasov, V. I., Gnezdilov, A. A., Gorgutsa, R. V., Grechnev, V. V., Denisov, Y. I., Dmitriev, A. V., Dryer, M., Yermolaev, Y. I., Eroshenko, E. A., Zherebtsov, G. A., Zhitnik, I. A., Zhukov, A. N., Zastenker, G. N., Zelenyi, L. M., Zeldovich, M. A., IvanovKholodnyi, G. S., Ignat'ev, A. P., Ishkov, V. N., Kolomiytsev, O. P., Krasheninnikov, I. A., Kudela, K., Kuzhevsky, B. M., Kuzin, S. V., Kuznetsov, V. D., Kuznetsov, S. N., Kurt, V. G., Lazutin, L. L., Leshchenko, L. N., Litvak, M. L., Logachev, Y. I., Lawrence, G., Markeev, A. K., Makhmutov, V. S., Mitrofanov, A. V., Mitrofanov, I. G., Morozov, O. V., Myagkova, I. N., Nusinov, A. A., Oparin, S. N., Panasenco, O. A., Pertsov, A. A., Petrukovich, A. A., Podorol'sky, A. N., Romashets, E. P., Svertilov, S. I., Svidsky, P. M., Svirzhevskaya, A. K., Svirzhevsky, N. S., Slemzin, V. A., Smith, Z., Sobel'man, I. I., Sobolev, D. E., Stozhkov, Y. I., Suvorova, A. V., Sukhodrev, N. K., Tindo, I. P., Tokhchukova, S. K., Fomichev, V. V., Chashey, I. V., Chertok, I. M., Shishov, V. I., Yushkov, B. Y., Yakovchouk, O. S., and Yanke, V. G.: Solar and Heliospheric Phenomena in OctoberNovember 2003: Causes and Effects, Cosmic Res., 42, 435-488, doi:10.1023/B:COSM.0000046229.24716.02, 2004.

Wang, Y. M., Ye, P., and Wang, S.: Multiple magnetic clouds: Several examples during March-April 2001, J. Geophys. Res., 108, 1370, doi:10.1029/2003JA009850, 2003.

Wanliss, J. A. and Showalter, K. M.: High-resolution global storm index: Dst versus SYM-H, J. Geophys. Res., 111, A02202, doi:10.1029/2005JA011034, 2006.

Wu, C.-C. and Lepping, R.: Effects of magnetic clouds on the occurrence of geomagnetic storms: The first 4 years of Wind, J. Geophys. Res., 107, 107, 1314, doi:10.1029/2001JA000161, 2002.

Yermolaev, Y. I., Yermolaev, M. Y., Lodkina, I. G., and Nikolaeva, N. S.: Statistical investigation of heliospheric conditions resulting in magnetic storms, Cosmic Res., 45, 1-8, doi:10.1134/S0010952507010017, 2007.

Yermolaev, Y. I., Nikolaeva, N. S., Lodkina, I. G., and Yermolaev, M. Y.: Specific interplanetary conditions for CIR-, Sheath-, and ICME-induced geomagnetic storms obtained by double superposed epoch analysis, Ann. Geophys., 28, 2177-2186, doi:10.5194/angeo-28-2177-2010, 2010.

Yermolaev, Y. I., Nikolaeva, N. S., Lodkina, I. G., and Yermolaev, M. Y.: Geoeffectiveness and efficiency of CIR, sheath, and ICME in generation of magnetic storms, J. Geophys. Res., 117, A00L07, doi:10.1029/2011JA017139, 2012.

Yermolaev, Y. I., Lodkina, I. G., Nikolaeva, N. S., and Yermolaev, M. Y.: Dynamics of large-scale solar wind streams ob- 
tained by the double superposed epoch analysis: dynamics of large solar wind streams, J. Geophys. Res., 120, 7094-7106, doi:10.1002/2015JA021274, 2015.

Zhang, J., Liemohn, M. W., Kozyra, J. U., Thomsen, M. F., Elliott, H. A., and Weygand, J. M.: A statistical comparison of solar wind sources of moderate and intense geomagnetic storms at solar minimum and maximum, J. Geophys. Res., 111, A01104, doi:10.1029/2005JA011065, 2006.
Zurbuchen, T. H. and Richardson, I. G.: In-Situ Solar Wind and Magnetic Field Signatures of Interplanetary Coronal Mass Ejections, Space Sci. Rev., 123, 31-43, doi:10.1007/s11214-0069010-4, 2006. 